\title{
Through the lens of work-integrated learning: Staff experiences of participating in person-centredness coach training in a Swedish hospital
}

\author{
Catrin E. Alverbratt*1 ${ }^{* 1}$ Charlotte T. Almkvist Hall ${ }^{2}$ \\ ${ }^{1}$ Department of Health Sciences, University West, Trollhattan, Sweden \\ ${ }^{2}$ Department of NU- Sjukvarden, Vastra Gotalandsregionen, Trollhattan, Sweden
}

Received: June 4, 2020

Accepted: August 26, 2020

Online Published: September 6, 2020

DOI: $10.5430 /$ jnep.v11n1p1

URL: https://doi.org/10.5430/jnep.v11n1p1

\begin{abstract}
Background/Objective: Over the past decade, many scientific articles have focused on the importance of person-centred care (or person centredness) in the health care sector. In practice, however, person centredness is difficult to operationalise. Thus, the role of "person-centredness coach" was created in a Swedish hospital to provide information, education, and reflection on person centredness. The aim was to describe this new role of a person-centeredness coach, and how the coaches experienced the development of a person-centred working method.

Methods: Qualitative semi-structured individual interviews were conducted with nine nursing staff. The data were analysed using inductive content analysis.

Results: The analysis resulted in three categories with seven subcategories: an eye opener (with the subcategories of a welcome change and person centredness throughout the organisation); an obstacle with potential (with the subcategories of theoretical vs. practical development of person centredness, difficulties in developing person centredness and proposals for promoting patient participation); and a challenging role (with the subcategories of necessary but a role that takes a long time to develop and the importance of favourable conditions).

Conclusions: The person-centredness coaches believed that the person-centred approach was important and that it should be the foundation of all care work within health care but, despite this, had difficulty in integrating person centredness into their practice. The person-centredness coaches found the coach training rewarding. They perceived that, from a learning perspective and through the lens of work-integrated learning, the results could be related to creating praxis, which may be seen as a development area for further research in operationalising person centredness.
\end{abstract}

Key Words: Education, Change, Learning, Person centredness, Work-integrated learning

\section{INTRODUCTION}

In the past decade, many scientific articles have addressed the topic of person-centred care (or person centredness), ${ }^{[1-5]}$ and studies have highlighted the importance of person-centred care (PCC) in health care and throughout the care chain. ${ }^{[6-8]}$
Despite this, the concept and meaning of PCC remain complicated. ${ }^{[9]}$ The concept itself is well known to nurses, but they find it difficult to operationalise and define it in practice. Researchers ${ }^{[9]}$ have argued that the concept needs a clear definition to be operationalised in practice. This could

\footnotetext{
*Correspondence: Catrin E. Alverbratt; Email: catrin.alverbratt@hv.se; Address: Department of Health Sciences, University West, 46186 Trollhattan, Sweden.
} 
reflect the findings of Alqahtani, Kitsantas and Rodans, ${ }^{[10]}$ who revealed that nurses were aware of and had positive attitudes towards the implementation of evidence-based practice, but those findings did not reflect the outcome scores of actually implemented evidence-based practice in the workplace. Van Achterberg, Schoonhoven and Gro ${ }^{[11]}$ highlight some general aspects of operationalising (or implementing) evidence-based methods, which involve resources, organisational routines, attitudes, influence and knowledge.

In regard to the aspect of knowledge, work-integrated learning (WIL) and learning-integrated work (LIW) ${ }^{[12,13]}$ are of interest in this area, with WIL being a general term describing the integration of theory and practice in a purposeful way. ${ }^{[12]}$ Van Rooijen ${ }^{[13]}$ explains that WIL is a prerequisite for LIW and that the synergy effects relate to lifelong learning. Gopee ${ }^{[14]}$ presents a model of lifelong learning in nurses, using a conceptual framework summarised by three factors-sociopolitical, individual/personal and organisational — that could be related to the concept of WIL. ${ }^{[12]}$

WIL is both similar to and different from learning at school and occurs both informally and formally, ${ }^{[12]}$ which are two perspectives on how learning occurs in workplaces. ${ }^{[15]}$ The other two perspectives are that workplace learning can be explored at diverse levels, either the individual level or that of larger networks and entire regions. According to a researcher, ${ }^{[15]}$ many factors influence the development of professional knowledge, and mentoring and coaching are mentioned as mediating tools for integrating theory and practice. Some of the other factors described include writing assignments, self-assessment and portfolios focusing on reflection. ${ }^{[16]}$ Coaching as a mediating tool can be examined in the context of a change project in a Swedish hospital in 2016. A Swedish health care region decided to allocate financial resources to the development of a more person-centred working method by adding change leaders, whose task was to train the nursing staff in person centring. The change leaders discovered a need related to disseminating knowledge about person centredness and understanding how nursing staff can translate action ethics into everyday work life. The aim in establishing the role of 'person-centredness coach' (PC coach) and educating nursing staff in both person centring and change management was to make the employees a resource in the development work of the managers of their wards and especially for all nursing in the wards in regard to informing, educating, and reflecting about the differences in working methods with and without a person-centred perspective.

The PC coach training was influenced by Gibbs' ${ }^{\text {' }}{ }^{[7]}$ reflective cycle and learning by doing (i.e., experiential learning), in which theory, practice, reflection, and action are closely linked and knowledge must be related to reality. ${ }^{[18]}$ Gibbs's reflective cycle, which consists of six stages (description, feelings, evaluation, analysis, conclusion and an action plan) was developed to give structure to experience-based learning. It offers a framework for examining experiences and, given its cyclic nature, lends itself particularly well to repeat experiences, allowing one to learn and plan from things that either did or did not go well. ${ }^{[17]}$ The aim of this study was to describe the new role of person-centeredness coaches, and how the coaches experienced the development of a person-centred working method.

\section{METHODS}

\subsection{Study design}

To determine how PC coaches described the new role of person-centredness coaches and how they experienced the development of a person-centred working method, a descriptive study was conducted based on qualitative data collected through semi-structured individual interviews. The data were analysed by inductive content analysis. ${ }^{[19]}$

\subsection{Context and setting}

An internal PC coach training divided into 10 sessions of two hours each was offered to nursing staff $(\mathrm{N}=56)$ at a Swedish hospital from January 2018 through September 2019. The change leader sent out information to all the ward managers through organisational channels. This was followed up by further contact with managers in a part of the organisation that has both psychiatric and medical wards. The managers were asked whether they wanted to participate, and those who were interested in joining subsequently selected the coaches by having a dialogue with interested employees.

These persons' names were then reported by the manager to the change leader, who sent out the course invitations. To gain a broad perspective, staff from both the psychiatric and medical wards were included. The time between the training sessions provided periods for reflection and for testing theoretical knowledge through course assignments in practical, everyday work. The results of the course assignments were shared via reflection during the following session, resulting in reflections and discussions such as, "Why do we do this in a certain situation, and can you do it differently?" Additionally, discussions about what characterises a good coach took place throughout the training as the coaches reflected on what had worked in the wards and what had seemed less effective. The coach training was planned and carried out for a long time, so the participants had time to slowly grow into the role and to reflect and test different approaches. ${ }^{[20]}$ After completing the coach training, the PC coaches became part of a network 
within a structure offering two administrative meetings each year to access continued support, to share experiences, to disseminate information and to embrace one another. A total of 56 nursing staff members underwent coach training from January 2018 through September 2019. Of those, 15 had either quit or changed jobs at the time of data collection. At the time of data collection, all nursing staff $(n=41)$ who had undergone coach training from January 2018 through May 2018 were informed about the study and invited to participate. Nursing staff who did not participate in the coach training were excluded.

\subsection{Participants}

A total of nine nursing staff members (two men and seven women), representing the psychiatric $(n=6)$ and medical ( $n$ =3) wards, participated in the study. The participants had worked in health care between 2 and 43 years.

\subsection{Data collection and analysis}

The data were collected through semi-structured interviews. To avoid influencing the participants, the request and the interviews were conducted by the first author (CA), who was not part of the coach training and who had never worked or had a close relationship with the participants. The nursing staff were asked whether they wanted to participate by means of an email with information about the study. Those who were interested in participating in an interview answered the email and were then contacted to schedule a time for the interview. The participants were asked to talk about the new role of PC coach and how the coaches experienced the development of a person-centred working method. The interviews were held in secluded rooms near the participants' workplaces from June 2018 through December 2018 and lasted between 16 and 41 minutes. They were recorded, subsequently transcribed verbatim and analysed using inductive content analysis. ${ }^{[19]}$ The analysis comprised several steps, starting with listening to the recording and reading the transcript several times to gain an understanding of the text. Next, the data were processed into meaning units, condensed and coded into categories and subcategories. ${ }^{[19]}$ To ensure credibility, the results are illustrated with quotations.

\subsection{Ethical considerations}

No ethical approval was required in accordance with Swedish ethical protocol, ${ }^{[21]}$ but, in accordance with ethical procedures stipulated by Swedish law ${ }^{[22]}$ and the Code of Ethics of the World Medical Association (Declaration of Helsinki), the participants received oral and written information about the study before it began and were informed that participation was voluntary. Written consent was submitted by those who wished to participate. The study was motivated by the fact that the results may produce new knowledge about the role of PC coaches and how they experience the development of a person-centred working method, but the questions in the interviews could arouse thoughts among the staff about not working enough with the coaching in their wards. However, that risk was considered to be small. The participants were allowed by managers at the hospital and in their wards to participate in the interviews during working hours, so they should have experienced no increased workload, but the staff who remained in the wards may have experienced an increased workload when the participants took part in the study. The risks of perceived stress in connection with participation were judged to be small in relation to the potential benefit of the study. The data and the participants' identities were treated confidentially; the participants' names and other identifying information were changed in the transcripts.

\section{Results}

The analysis produced three categories and seven subcategories. The categories that emerged were: an eye opener (with the subcategories of a welcome change and person centredness throughout the organisation); an obstacle with potential (with the subcategories of theoretical vs. practical development of person centredness, difficulties in developing person centredness and proposals for promoting patient participation); and a challenging role (including the subcategories of necessary but a role that takes a long time to develop and the importance of favourable conditions). An overview of the results is given in Table 1.

Table 1. Overview of the results

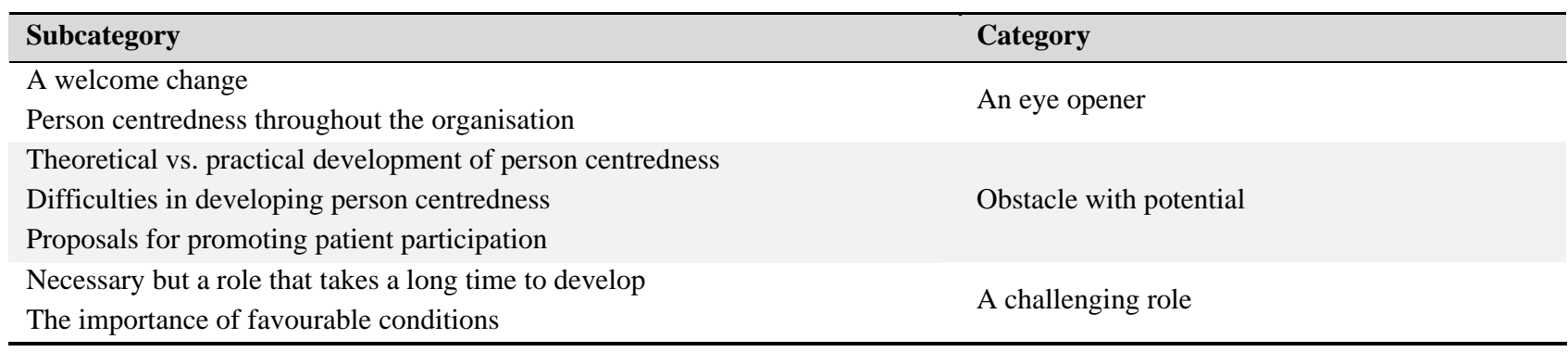




\subsection{An eye opener}

The category an eye opener and the related subcategories a welcome change and person centredness throughout the organisation relate to the coaches' realising the need to change their working methods for the organisation to meet current and future requirements.

\subsubsection{A welcome change}

The PC coaches thought that the coach training was good when discussions and reflections with other participants were highlighted. They found these to be particularly rewarding. Although the PC coaches felt that the training did not contain anything really "new", they emphasised that their education contributed to a change in their attitudes and that they now thought more about asking open questions and understanding an individual's resources, which contributed to beneficial meetings with patients. They saw the person-centred working method as being all pervasive, and most of the coaches emphasised that the person-centred working method accorded with their views on how to work in Swedish health care.

"This person-centred way of working, I feel that it fits very well with what I believe in; I think that's how we should work" (interview 7).

The PC coaches said that the reactions of the majority of their colleagues concurred with their own reactions in that colleagues who had received information about PCC thought of it as something positive that was needed and welcomed.

\subsubsection{Person-centredness throughout the organisation}

To sustain employees, the PC coaches emphasised the importance of also taking a person-centred approach towards one another and that it is important to see all the individuals in the organisation as people with needs and resources. One example mentioned seeing all colleagues as being valuable, even those who might be perceived as difficult to work with.

"The fact is that colleagues who are difficult to work with also add something positive to the department" (interview 9).

The coaches also emphasised the importance of the whole organisation's being person centred and that it should not be only a decision of top management. Regardless of where one is in the organisation, it is important that all see one another as persons, not as roles, for the ethics of action to be observed throughout the organisation. The participants gave examples and stated that this should be clear in the advertising and recruitment of managers.

\subsection{Obstacle with potential}

The category obstacle with potential and the associated subcategories (theoretical vs practical development of PCC, difficulties in developing PCC and proposals for promoting patient participation) concerned the theoretical and practical application of PCC in health care work, where the coaches were in different phases. The coaches emphasised that existing routines and systems were not flexible enough to handle a more person-centred approach. The process of developing a more person-centred working method heightened awareness of the emergence and existence of rules and routines. It also created an awareness of the need to review information and materials and to further develop this from a user perspective.

\subsubsection{Theoretical vs. practical development of person cen- tredness}

Based on organisational conditions, the coaches argued that they were in different phases of developing a more personcentred working method. A majority of the coaches stated that they had not yet begun to work practically with a personcentred approach in their wards. Theoretically, however, the work of informing colleagues, preferably in conjunction with the workplace meetings, had begun for a majority of the coaches. Many coaches had also discussed person centredness in their workplace workshops or planned to use the next workplace workshop to talk about person centredness in order to reach all employees.

"I have not started yet, but I have provided information about person centredness at our workplace meeting-what it is-and that we will have study circles for the staff" (interview 5).

However, some of the coaches stated that their departments had started to work with a more person-centred approach. When colleagues had been informed, when the workplace workshop during the past year had been devoted to person centredness (PC) and when there were regular weekly staff meetings, they were able to work on diverse areas to make nursing care more person centred. However, the problem of finding time to convert theory into practice remained.

\subsubsection{Difficulties in developing person centredness}

A problem that raised an obstacle to the person-centred working method was the current system of documenting agreements and plans. Documentation differs depending on where one works, which makes it difficult to apply a person-centred approach. In connection with the documentation's obstacles and difficulties, the coaches also mentioned care plans, which have an overall structure. Changing the conditions of the care plans was considered to be too labour intensive to fall within the coaches' mission and role. Despite this, the work of documenting according to a person-centred working method was ongoing at one of the wards, where an evaluation date had been set.

"We cannot change anything; for example, we do not get our 
own care plan for outpatient care. The overall care plan is a big job; I had wished we had one for just the care session, but we didn't get that. It feels a little gritty because it's so much stuff" (interview 3).

The coaches also highlighted that they wanted more support and hands-on tips, templates and routines for what to request and how to document according to PC in the existing documentation systems. The desire for templates and guidelines also applied to other parts of health care in promoting the development of person centredness.

\subsubsection{Proposals for promoting patient participation}

A direct consequence of the introduction of a more personcentred approach was that one department changed its existing routines, and some routines and regulations were removed to benefit the patients. An example of this is that patients are now served coffee between the hours of 06:00 and 18:00 whereas, previously, coffee had been served only with meals. The change did not result in patients drinking more coffee; instead, the craving for coffee lessened in the department. This was only one example of a person-centred activity's being implemented, and the coaches revealed that there is much left to do.

"We have some rules here, rules that we can argue for because we have removed some routines that we couldn't really explain why we had, which is also a part of PC. Why do we really have these rules?" (interview 2 ).

The coaches shared many ideas about areas needing development; for example, informational brochures, signs and forms should be reviewed to promote patient participation. The medical round was another activity that several of the coaches highlighted as an area needing more work. For example, in psychiatry, the patients do not participate, which is an area for development. The coaches representing the medical wards also highlighted the need to change the medical round structure to further develop patient participation. A general conclusion that emerged from the coaches was that one should want to work for patient participation as the coaches had noticed several benefits for the patients in the care process. The coaches revealed that one advantage of PC is that it allows one to try out and evaluate different ways of working.

\subsection{A role with challenges}

The category a role with challenges and the associated subcategories (necessary but a role that takes a long time to develop and the importance of favourable conditions) concerned the role of being a PC coach, which was considered important but still in its development phase. The coaches also emphasised the importance of not being alone as a coach and the value of a supportive, responsible manager. The coaches also emphasised the importance of all health care professionals' developing a person-centred approach.

\subsubsection{Necessary but a role that takes a long time to develop}

Before the coach training, the coaches considered it a challenging role, and, after the training, they were still uncertain as to how to perform the role in their wards. The coaches expressed that they must work educationally and psychologically to inspire without being a teacher. The coaches saw great importance in concretising PC in care work to make it easier for their colleagues. The coaches also emphasised that it was not they who would be responsible for all the PC work in the ward because the transition to PC is a shared responsibility.

"In some situations, you must be able to take control, however, with caution; otherwise, your work is to encourage what works and try to correct things that do not work well" (interview 9).

The coaches considered the role of coach valuable and thought that coaches were needed. One key characteristic is that a coach should be a person who enjoys change projects. They expressed the importance of long-term work and emphasised that the transition to a person-centred approach should and must take time.

\subsubsection{The importance of favourable conditions}

The coaches considered it important that there be several coaches in the same ward as they considered the workload to be too heavy for one person. They suggested that there should be at least one nurse and one nurse assistant in each ward (and preferably more people) to meet the emerging needs in the transition to a more person-centred way of working.

"If you send away a person who is supposed to [implement] a completely new approach at their ward, I feel that you might as well give up. It's not fair to that person" (interview 2).

The coaches also emphasised the importance of a supportive manager in that the main responsibility for the development of PC belongs to the manager of the ward. The coaches also expressed a concern for situations in which they were the only coach in the ward; if the coach and the manager are both responsible for introducing PC in the ward, this could result in an asymmetrical relationship between them. The coaches also emphasised the importance of including other occupational categories when developing a person-centred approach because an all-pervasive approach such as person centredness cannot be handled by nursing staff alone. 


\section{Discussion}

The present study aimed to describe the new role of PC coaches and how they experienced the development of a person-centred working method. This resulted in three categories and seven subcategories: an eye opener (a welcome change; person centredness throughout the organisation), obstacle with potential (theoretical vs. practical development of person centredness; difficulties in developing person centredness; proposals for promoting patient participation) and a challenging role (necessary but a role that takes a long time to develop; the importance of favourable conditions). The results reveal that coach training is a rewarding experience that contributes to a change of mindset ${ }^{[1]}$ even though the PC coaches were in different phases of developing a personcentred approach in practice. Theoretically, all coaches applied a person-centred approach, which can be compared to Backman et al. ${ }^{[1]}$ who, focusing on the perspective of health care managers, stated that in order to be person-centred, it is not enough to just have a person-centred mindset, a mindset based on reflection, but it also requires integrating and maintaining person-centeredness in practice, and this last step was difficult. From a learning perspective, through the lenses of WIL and LIW, ${ }^{[12,13]}$ this result could also be related to the process of creating praxis. Praxis results from the development of metacognitive abilities that allow students to test their experiences and is defined as the ability to combine practical and theoretical knowledge and reflection. ${ }^{[23]}$ The results of this study reveal that PC coaches succeeded in gaining reflective and theoretical knowledge about PC, but the ability to combine these components with practical knowledge had not been achieved at the time of data collection. The results indicate that the coach role is challenging as the coaches were convinced that they had to work educationally and psychologically with their colleagues to foster a PC approach in their wards. This could be considered a future research area in terms of WIL and the operationalisation of $\mathrm{PC}$ in practice.

The results show that PC coaches should not be responsible for all PC work in a ward because the transition to PC is a shared responsibility. ${ }^{[24]}$ The PC coaches emphasised that existing routines and documentation systems are not flexible enough to meet a more person-centred approach, ${ }^{[25]}$ which is in line with Moore et al., ${ }^{[26]}$ who emphasise the importance of strong leadership in overcoming barriers (such as existing documentation systems). Among several facilitators, the researchers ${ }^{[26]}$ also emphasise strong leadership in the successful implementation of PC. Another facilitating factor was training, which reflects the focus of the present study and the importance of WIL. Wolf, Ulin and Carlström ${ }^{[27]}$ argue that the implementation of PCC affects organisational culture, but it requires time, practice and dedicated staff, which is in line with the results of this study, in which the PC coaches highlighted time as an important factor in developing person centredness in health care. The aspect of dedicated staff ${ }^{[27]}$ can be related to the PC coaches themselves, who considered that coaches should have the characteristic of enjoying change projects. This could be seen as a prerequisite in change management. Whether there will be a change in the organisational culture in favour of a person-centred approach at the present hospital remains to be seen, which may be a suggestion for future research.

\section{Limitations}

The data were collected from only one hospital in Sweden, which is a limitation. Another limitation is the low number of participating coaches in the study; only nine PC coaches representing eight wards participated in this study (six from psychiatric and three from medical wards). This may be because the data collection took place as the hospital experienced large financial cuts, which may have affected the participants' ability to participate in the interview during working hours. Also, due to the hospital's economic situation, no dropout analysis was conducted in the study. The distribution between psychiatric and medical wards is a limitation as the cohort could have been more evenly distributed. The transferability of the results is another limitation as it is not possible to generalise the findings outside the studied group. However, the result showing a mindset of person centredness among the PC coaches is supported by other studies..$^{[1,9,10]}$

\section{Conclusion}

PC coaches considered the person-centred approach important and thought that it should constitute the foundation of all care work within health care. Despite this, the coaches had difficulties in integrating PC into practice. The PC coaches found the coach training rewarding, and, when seen from a learning perspective through the lens of WIL, these results could relate to the process of creating praxis, which can be seen as a development area for further research in operationalising $\mathrm{PC}$ in practice.

\section{ACKNOWLEDGEMENTS}

The study was partially supported by the Research and Development Council-Fyrbodal Sweden. The authors would like to thank all the PC coaches and the participating hospital for their participation in the study.

\section{CONFlicts of INTEREST Disclosure}

The authors declare that they have no conflicts of interest. 


\section{REFERENCES}

[1] Backman A, Ahnlund P, Sjögren K, et al. Embodying person-centred being and doing: Leading towards person-centred care in nursing homes as narrated by managers. Journal of Clinical Nursing. 2020; 29(1-2): 172-83. PMid:31612556 https://doi.org/10.1111/ jocn. 15075

[2] Ekman I, Swedberg K, Taft C, et al. Person-centered care-ready for prime time. European Journal of Cardiovascular Nursing. 2011; 10(4): 248-251. PMid:21764386 https : //doi.org/10.1016/j. ejcnurse. 2011.06 .008

[3] McCormack B, McCance T. Person-centred Nursing: Theory, models and methods. Oxford: Blackwell Publishing; 2010. https: //doi.org/10.1002/9781444390506

[4] McCance T, Gribben B, McCormack B, Laird EA. Promoting personcentred practice within acute care: the impact of culture and context on a facilitated practice development programme. International Practice Development Journal. 2013; 3(1).

[5] Edvardsson D. Notes on person-centred care: What it is and what it is not. SAGE Publications Sage UK: London, England; 2015. https://doi.org/10.1177/0107408315582296

[6] Fors A, Ekman I, Taft C, et al. Person-centred care after acute coronary syndrome, from hospital to primary care-a randomised controlled trial. International Journal of Cardiology. 2015; 187: 6939. PMid:25919754 https ://doi.org/10.1016/j.ijcard. 201 5.03 .336

[7] Fors A, Swedberg K, Ulin K, et al. Effects of person-centred care after an event of acute coronary syndrome: two-year follow-up of a randomised controlled trial. International Journal of Cardiology. 2017; 249(42): 7. PMid:28893432 https ://doi.org/10.1016/ j.ijcard. 2017.08.069

[8] Jakobsson S, Ringström G, Andersson E, et al. Patient safety before and after implementing person-centred inpatient care-A quasiexperimental study. Journal of Clinical Nursing. 2020; 29(3-4): 60212. PMid:31769572 https://doi.org/10.1111/jocn. 15120

[9] Byrne AL, Baldwin A, Harvey C. Whose centre is it anyway? Defining person-centred care in nursing: An integrative review. PloS One. 2020; 15(3): e0229923. PMid:32155182 https ://doi .org/10.1 371/journal. pone. 0229923

[10] Alqahtani N, Oh M, Kitsantas P, et al. Nurses' evidence-based practice knowledge, attitudes and implementation: A crosssectional study. Journal of Clinical Nursing. 2020; 29(1-2): 274-83. PMid:31714647 https ://doi.org/10.1111/jocn. 15097

[11] Van Achterberg T, Schoonhoven L, Grol R. Nursing implementation science: how evidence-based nursing requires evidence-based implementation. Journal of Nursing Scholarship. 2008; 40(4): 302-10. PMid:19094144 https ://doi.org/10.1111/j.1547-5069. 20 08.00243.x

[12] Patrick CJ, Peach D, Pocknee C, et al. The WIL (Work Integrated Learning) report: A national scoping study: Queensland University of Technology; 2008.

[13] Van Rooijen DM. Transforming 21st century corporate-university engagement: From work-integrated learning (WIL) to learning- integrated work (LIW). An International Journal for Collaborative/work Integrated Learning. 2011; 45(1): 5-10.

[14] Gopee N. Facilitating the implementation of lifelong learning in nursing. British Journal of Nursing. 2005; 14(14): 761-7. PMid:16116381 https://doi.org/10.12968/bjon.2005.14.14.18553

[15] Tynjälä P. Perspectives into learning at the workplace. Educational research review. 2008; 3(2): 130-54. https://doi.org/10.101 $6 / j$.edurev. 2007.12.001

[16] Pennbrant S, Nunstedt H. The work-integrated learning combined with the portfolio method-A pedagogical strategy and tool in nursing education for developing professional competence. Journal of Nursing Education and Practice. 2017; 8(2): 8-15. https://doi .org/ 10.5430/jnep.v8n2p8

[17] Gibbs G. The reflective cycle, Further Education Unit, Oxford Polytechnic, Oxford. 1988.

[18] Ord J. John Dewey and Experiential Learning: Developing the theory of youth work. Youth \& Policy. 2012; 108(1): 55-72.

[19] Graneheim U, Lundman B. Qualitative content analysis in nursing research: concepts, procedures and measures to achieve trustworthiness. Nurse education today. 2004; 24(2): 105-12. PMid:14769454 https://doi.org/10.1016/j.nedt.2003.10.001

[20] Smith K. Reflection and person-centredness in practice development. International Practice Development Journal. 2016; 6(1). https : //doi.org/10.19043/ipdj.61.012

[21] Swedish Research Council. Good research practice. 2017. Sweden: Stockholm. Available from: https: //www.vr.se/download/18.5639980c162791bbfe697882/1 $555334908942 /$ Good-Research-Practice_VR_2017.pdf

[22] SFS 2003:460. Law 2003:460 about the ethical review of research involving humans. 2003. Stockholm: Riksdagen.

[23] Lyckhage E, Pennbrant S. Work-integrated learning: A didactic tool to develop praxis in nurse education. Advances in Nursing Science. 2014; 37(1): 61-9. PMid:24469089 https ://doi.org/10.1097/ ANS 0000000000000018

[24] The Swedish Association of Local Authorities and Regions. Personcentrerad vård i Sverige. [A survey of person centred care in Sweden]. 2018. Stockholm: Sweden. Avaliable from www.skl.se. ISBN-number: 978-91-7585-631-5. Available from: https://webbutik.skr.se/bilder/artiklar/pdf/75 85-631-5.pdf?issuusl=ignore

[25] Rosengren K, Ulin K, Carlström E. Characteristic of person-centered care as documented in medical records at a medical department-A mixed methods. Journal of Hospital Administration. 2019; 8(2). https://doi.org/10.5430/jha.v8n2p7

[26] Moore L, Britten N, Lydahl D, et al. Barriers and facilitators to the implementation of person-centred care in different healthcare contexts. Scandinavian Journal of Caring Sciences. 2017; 31(4): 662-73. PMid:27859459 https : //doi.org/10.1111/scs . 12376

[27] Wolf A, Ulin K, Carlström E. Changing the wardculture in a clinic during the implementation of person-centred care. Journal of Hospital Administration. 2017; 6(5): 31-39. https ://doi .org/10.543 0/jha.v6n5p31 